\title{
The Broadening Scope of Validation: Towards Best Practices in the World of Bioanalysis
}



\author{
European Bioanalysis Forum, 2nd Annual Open Symposium \\ Hisperia Towers, Barcelona, Spain, 2-4 December 2009
}

The European Bioanalysis Forum is a bioanalytical discussion group comprised of European pharmaceutical companies (27 members to date). The membership share a common vision to advance the shared understanding of topical concerns through discussion of scientific, technological and regulatory issues of bioanalytical interest. The 2 nd Annual Open Symposium was much anticipated after the success of the first one. The symposium included sessions on the European Medicines Agency draft bioanalytical guidance, challenges in the analysis and validation of biomarkers, metabolite quantification issues, dried blood spot technology, the analysis of proteins and peptides by MS, latest hot topics in ligand-binding assays, unresolved issues in method validation and a plenary session from the Platinum sponsors. Experts and key opinion leaders were invited as guest speakers. A total of 420 delegates (up from 251 delegates in 2008) attended from 160 organizations, representing a large percentage of the European and international bioanalytical community. In addition to 40 oral presentations, 72 posters were presented and there was a vendor exposition of 35 companies.

\section{Towards European Medicines Agency guidelines for Bioanalysis}

The US FDA Guidance on Bioanalytical Method Validation has become the primary reference document in bioanalytical laboratories performing regulatory analyses in support of new product submissions [101]. However, in 2008, the European Medicines Agency (EMA) produced a concept paper on Bioanalytical Method Validation [102], with a draft document available for review in December 2009 [103]. The fear of many working in the field was that two guidance documents with contradictory content would emerge, causing much headache and additional challenges. It was with this background that Jan Welink (EMA and Dutch Medicines Evaluation Board) opened the conference with a presentation on the EMA draft guidance document. Their idea is to incorporate the FDA and American Association of Pharmaceutical Scientists (AAPS) recent thinking into an EMA guideline and not to invent a totally new guideline. The structure of the guideline will include sections on method validation, study sample analysis, incurred sample reproducibility (ISR) and study report content. The draft guideline is open for comment for the next 6 months. The detailed content seems aligned with the current FDA guidance, with the inclusion of additional clarity on specific topics. Olivier Le Blaye (French Health Products Safety Agency and an author of the EMA guidance) presented feedback from various French and EU inspections that had influenced some of the content of the draft EMA document. He focused on four areas, all relating to chromatographic methods:

- Internal standard response variation;

- Anticoagulant choice and consistency;

- Definition of an analytical run;

- Extension of ISR investigation to include all samples showing large differences even if $67 \%$ of the samples reanalyzed meet the $20 \%$ acceptance criteria.

Steve Lowes (Advion, representing the AAPS Bioanalytical Focus Group) made a plea for consistency in the evolution of regulated bioanalysis and recommended an ICH guideline generated through collaboration between the FDA and EMA. He pointed out that after 20 years of discussion, there is still room for further optimization of the guidelines as several topics - for instance, internal standard response acceptance criteria, method robustness, manual integration and multi-analyte data acceptance - still have no consensus. In addition, new technologies, strategies and analytes mean that the end point is constantly changing. In a global economy, as we submit data to various global agencies, a single ICH guideline would be the preferable outcome. Harmonization was the common speaker theme and this was reflected in the presentation from Dr Viswanathan (FDA). He mentioned the global benefit from a single, unified, global guidance. He emphasized the need to move

\section{Richard W Abbott \\ Department of Biosciences, \\ Shire Pharmaceuticals, \\ Hampshire International \\ Business Park, Basingstoke, \\ Hampshire, RG24 8EP, UK \\ Tel.: +44 I25 6894575 \\ Fax: +44I256894703 \\ E-mail: rabbott@shire.com}


forward through international harmonization, although he did state that the FDA are working on an updated guidance document of their own. Nevertheless, he would like to move towards a unified international standard, with more regular meetings to develop and understand the present guidelines, as well as to agree collectively on new areas for discussion. Finally in this session, Berthold Lausecker (Roche, representing the European Bioanalysis Forum [EBF]) presented the consolidated response of the EBF member companies to the EMA concept paper. In general, the EBF supports the EMA guideline as a step towards further international harmonization in bioanalysis. Specific points of concern were highlighted and hopefully the draft EMA guidance will reflect these concerns. The EBF would like to see a guidance document on biomarker assay validation and analysis but acknowledges that this would not be manageable with the current guidance. A panel discussion concluded the session. It was refreshing to hear agreement that a harmonized guideline is the way forward. The EMA will introduce their guidance as a first step, as it will represent the agreement of 27 member countries. The FDA is willing to work with the EMA and the wider international community and an ICH committee should be instituted. It was acknowledged that bioanalytical forums, such as the EBF and Bioanalytical Focus Group (AAPS BFG), can help to influence the regulatory authorities. It is envisioned that a further harmonized document on biotechnology will follow later. To facilitate further discussion on the EMA draft bioanalytical method validation guidance document, the EBF is jointly sponsoring a meeting with the European Federation for Pharmaceutical Sciences (EUFEPS) in Brussels (15-16 April 2010) [104].

\section{Biomarkers: validation \& analytical challenges}

Ron Bowsher (Millipore) opened this session with a comprehensive overview of the challenges around analytical validation of commercial assay kits for novel biomarkers. This is an extremely complex subject and the three types of formats are all less than ideal - pharmaceutical industrydeveloped assays often lack robustness, clinicalgrade diagnostic kits are FDA-approved and CLIA regulated but are not intended to support drug development, and research-grade diagnostic kits are more suitable but there are problems in meeting GLP standards with reference material quality, kit components, kit validation and operational use. Kit-performance criteria often refer to LOD versus LLOQ. As we are aware, LOD is not a term used in regulatory bioanalysis. Clearly kits must be validated 'for the conditions of intended use' and a multiplexing 'fit-for-purpose' strategy is recommended to maximize efficiency and cost effectiveness. Stephan Mueller (Roche) presented on the subject of MS and NMR-based small-molecule mechanistic biomarker applications in discovery. The diversity of the analytes encountered requires a wide variety of techniques to be available and the endogenous nature of the analytes results in greater complexity regarding calculation and interpretation of data. Chris Beaver (MDS) gave a contract research organization (CRO) perspective on the challenges in adapting commercial kits for regulated bioanalysis. Clear processes for the qualification of components are essential and it is important to work closely with kit vendors to understand their quality-control processes and ensure supply for the duration of the clinical program. Finally in this session, a central laboratory view was provided by Claire Huguet (Quintiles). Customers are looking for qualified biomarker measurements following regulatory validation guidelines and expecting high-throughput analyses in multiple, global studies of long duration. Assay development is tough and there are frequent issues with study plans and reality, as well as a need to qualify multiple laboratories. Technical issues include the availability and stability of appropriate calibration materials, having to decrease the LOD of a standardized kit and reproducibility of identical materials.

\section{Challenges in \\ metabolite quantification}

Philip Timmerman (Johnson and Johnson, representing the EBF) provided feedback on the EBF discussions on metabolite quantification. This is complex and occurs at all stages of development to different quality standards. Moreover, there has been an increase in the number of metabolites being quantified since the issue of the metabolites in safety testing (MIST) guidance [105]. Analytical challenges include differences in instrument response between metabolites, matrix issues and stability problems. In order to develop effective approaches, there is a need to better understand the terms 'tiered' approach and 'qualified' assay and, so, move towards a harmonization of strategies within the industry. Following an EBF questionnaire, the member companies were found to be aligned on $80 \%$ of topics and a White Paper will be 
published in 2010. This will include a number of recommendations, but the key point will be the need to differentiate between the degree and appropriateness of validation required, depending on the development stage of the project. Screening, qualified and validated assays should be utilized as appropriate and the EBF recommends specific context for the use of each type of assay, which will be documented in the White Paper. Glen Hawthorne (Astra-Zeneca) reviewed the approach to the MIST guidelines at AstraZeneca. In summary, Astra-Zeneca retain samples from their repeat-dose toxicology studies so that they can be compared against the human steady-state samples (multiple ascending dose study) to check that metabolites are comparable. If the results indicate a unique or disproportionate metabolite then this is quantified. However, there are significant logistical, technical and procedural challenges involved in arriving at the required end point. Fabio Garofolo (Algorithme Pharma) looked at the use of ISR to evaluate the possible impact of unstable metabolites on data generated in regulated bioanalysis with emphasis on the potential for metabolite conversion during the sample-extraction process for $N$-oxides, lactones and glucuronides (especially acyl glucuronides).

\section{Platinum sponsor session}

This session covered a range of topics, reflecting the mixed interests of the conference Platinum sponsors, which included CROs and instrument vendors.

Gerd Paulus (SwissBioAnalytics) presented their interpretation of the MIST guidance and the strategy and workflows used at SwissBioAnalytics in human radiolabeled absorption, distribution, metabolism and excretion studies. The need for earlier characterization and quantitation is apparent, as is the need to recognize the different skillsets required in metabolism and bioanalysis, with optimum resolution of the issues being facilitated by the degree of interaction and positive communication between the two groups. Martin Nemansky (PRA International) switched the subject to flow cytometry, the measurement and characterization of the physical and chemical characteristics of cells in a fluid stream by optical means - light scattering, light excitation and emission of fluorochrome particles. As a result, safety and efficacy can be assessed directly at a cellular level. Phenotyping, functional assays and occupancy assays are common applications. In terms of validation, there is no comprehensive guidance (yet) but it is recommended that the assays should be shown to be fit-for-purpose, following similar criteria to the regular bioanalytical guidance.

Rob Plumb (Waters) looked at the bioanalytical challenge of high-sensitivity separations combined with the requirement to improve productivity. Through the Waters platform, he described how the business requirements of continued laboratory success and productivity gains can be achieved. Finally, in this session, Yves Le Blanc (MDS) took a look into the future possibilities for MS design that would allow optimized qualitative and quantitative analysis. Multiple manufacturers have shown recent advances in high-resolution MS that allow for resolution greater than 30,000 with a linear dynamic range in excess of four orders of magnitude and acquisition rates greater than $10 \mathrm{spectra} / \mathrm{s}$, and have raised the possibility of high-resolution, single-stage MS (TOF) for quantitative/qualitative bioanalysis.

\section{Novel approaches for the analysis of proteins \& peptides by MS technology}

This area has become a hot topic in the past couple of years, as the number of peptide/protein drugs in development has increased and companies are looking for novel approaches to enable fast method development. MS offers a rapid and selective alternative to ligand-binding assays (LBAs; in particular the 6-9 months required to raise antibodies). The theme of the presentation by Yves Le Blanc was peptide quantitation using the novel approach of targeted-enhanced multiply charged scanning (t-EMC), as opposed to multiple reaction monitoring (MRM). t-EMC relies on selective discrimination of singly charged noise prior to mass analysis in a hybrid quadrupole linear ion trap (QqLIT). It provides comparable selectivity to MRM without the fragmentation (and multiply charged ions) and associated laborious instrument tuning. It also encompasses the phenomenon of combining MRM transitions to improve precision. The only required information is the $\mathrm{m} / \mathrm{z}$ of the peptide analyzed, which can easily be predicted from the amino acid sequence. Lieve Dillen (Johnson and Johnson) described the use of LC-MS/MS to measure amyloid peptides in cerebrospinal fluid (CSF) as biomarkers for Alzheimer's disease. Previously, immunological assays have given variable results. An API $4000^{\mathrm{TM}}$ has been employed for A $\beta 1-38$, $A \beta 1-40$ and $A \beta 1-42$ amyloid peptide-screening assays using negative-ion electrospray. Excellent assay performance was demonstrated (LLOQ $0.5 \mathrm{ng} / \mathrm{ml}$ for all peptides), although different 
matrix effects between simulated CSF and dog CSF have proven difficult to understand and so relative quantitative data (screening) have only been reported thus far for $A \beta 1-38$ and $A \beta 1-42$. The final goal is absolute quantification (qualified assay). Rohan Thakur (Taylor Technology) presented on the impact of endogenous phospholipids on the ion suppression of multiply charged compounds, such as peptides and proteins, which has yet to be fully characterized, despite having been studied extensively for singly charged small molecules. Example molecules chosen for this study included angiotensin 1 (charge state $[\mathrm{CS}]+2)$, oxytocin $(\mathrm{CS}+2)$, vasopressin $(\mathrm{CS}+2)$ and apomyoglobin $(C S+20)$ in various extracts of different plasma matrices. Multiply charged compounds seem to show a matrix enhancement compared with suppression with singly charged compounds, although this may be related to the 'surfactant' nature of hydrophobic peptides rather than multiply charged states. Michail Alterman (FDA - Center for Biologics Evaluation and Research) reviewed the application of MS-based proteomics in influenza virus research and vaccine manufacturing. His group have shown that such methods can be used to distinguish different influenza types and variants and determine the relative quantities of comigrating proteins using influenza virus preparations. In addition, other viral and host cell proteins have been identified, making this a perfect method to characterize vaccine preparations. Together with modern techniques to quantify the antigens and evaluate antigenic structure, the MS-based analysis will provide quality control of the final product that is distributed for human use. Completing this session, Yasser Ismail (Waters) described their approach to bioanalytical method development for therapeutic peptides. A total of 12 therapeutic peptides with a broad range of properties were selected for this work. The use of mixed-mode ion exchange $\mu$-elution SPE coupled with UPLC, a sensitive triple quadrupole MS system and simple protocols have been shown to simplify the method-development process for peptide therapeutics. Using these approaches, linearity over four orders of magnitude and an LLOQ of approximately $20 \mathrm{pg} / \mathrm{ml}$ were also demonstrated for a key peptide, desmopressin.

\section{Dried blood spots}

This technique, in use for 40 years but only recently applied to bioanalysis, has become a very hot topic in the past couple of years. Neil Spooner (GlaxoSmithKline [GSK]) introduced the topic by presenting the implementation of the technique at GSK and looking at future directions. He reviewed the numerous advantages of the low blood volume technique, including the benefits relating to animal reduction, data quality, improved patient recruitment (especially in paediatric studies), study cost reduction, simplified sample processing, the potential to minimize instability issues for labile molecules and reduced shipping and storage costs. Despite all the advantages, life is more difficult for the bioanalyst with sensitivity issues to consider owing to the reduced blood volume as well as the manual nature of the technique to date. Work is ongoing at GSK with a number of technologies and partners to address these issues. Marie-Claude Fraschini (SanofiAventis) presented the Sanofi-Aventis perspective on dried blood spots (DBS). An antimalarial project using DBS has been running in Africa for a few years. A DBS strategy has been agreed globally with feasibility testing over the past few months. A total of eight out of 34 compounds tested failed, all due to sensitivity issues. Some interesting positive findings include 24-h stability of a compound on DBS at room temperature compared with hydrolytic conversion of $64 \%$ of the compound to a metabolite after $4 \mathrm{~h}$ at room temperature in a conventional whole-blood sample. Sanofi-Aventis is also looking at automation and instrumentation sensitivity improvements as well as metabolite ID and biotherapeutic applications. Chris Smith (Astra-Zeneca) continued the session with the Astra-Zeneca strategy on DBS. Their reasons for investigating the technique are very similar to those of GSK and Sanofi-Aventis. They have compared DBS, whole-blood microsampling and plasma samples for toxicokinetic (TK) studies in rat and mouse. Whole-blood microsampling and DBS samples were found to produce identical TK results. In the future they will be investigating the use of DBS to solve prodrug instability issues, as well as looking at sensitivity issues and metabonomics applications. Lee Goodwin (Covance) described their investigation into the sensitivity limitations of DBS applications. He used pioglitazone as an example (LLOQ: $5 \mathrm{ng} / \mathrm{ml}$ for a rat DBS assay) but demonstrated that with appropriate adjustments in sample volume and extraction and instrument choice that it is possible to reach a LLOQ of $5 \mathrm{pg} / \mathrm{ml}$. Whole-blood and DBS samples again showed superimposable results. Impressive ISR results were also presented with the comparison made between two different laboratories. Finally, Dieter Zimmer (Harlan) suggested that DBS is a revolution similar to 
LC-API/MS approximately 20 years ago. His experimental work included the testing of ten analytes, using a generic extraction solvent mixture and suggested that the internal standard should be spotted onto the card prior to the blood sample. He agreed that bioanalytical method development is more difficult but fully justified by the benefits. He hopes to find an automation solution in the future and that the cost of the DBS cards will decrease over time.

The session concluded with a panel discussion. There was agreement that the practical aspects of the bioanalytical approach are well advanced and the technique has been shown to be very useful with many advantages. Further development of the DBS approach now depends on the attitude of the customer (toxicologists, pharmacokineticists, clinical development teams and sample logistics management) and the regulators. To facilitate this discussion, the EBF is sponsoring a meeting in Brussels, to be held on 17-18 June 2010 [106].

\section{Latest developments \& current hot topics in LBAs}

Claudia Berger (Solvay) opened this session on the subject of preclinical strategy and immunogenicity risk assessment for oral recombinant enzyme-replacement therapy. The focus of this talk was recombinant lipase therapy for the treatment of pancreatic exocrine insufficiency (PEI). Proteins are generally degraded in the GI tract and absorbed as peptides or amino acids. The bioanalytical assay for assessing pharmacokinetics (PK) was a sandwich ELISA, which was complicated as the compound had a very short half-life (a few minutes), necessitating an assay with a sensitivity of at least $0.00004 \%$ of the dose in preclinical studies. As a low-risk biological the overall strategy for recombinant lipase included an immunogenicity program run in parallel with Phase I and II studies. The next speaker, Paulina Rakowska (National Physical Laboratory, UK) discussed standard curves for immunoassay data analysis, as there is no overall agreement on the choice of calibration model to fit immunoassay data. The 4-parameter logistic (PL) and 5-PL models work well for many immunoassays but with some exceptions. Cubic spline models were found to be very flexible compared with other functions, can adapt to data with almost any characteristic and were found to have increased dynamic range. Inge Dreher (Abbott) focused on cell-based assays for neutralizing antidrug antibodies (ADAs). At Abbott, the TK profile together with an ADA-screening assay are used to assess neutralizing antibody incidence for preclinical studies, but in the clinical phase, development and validation of cell-based neutralizing assays must be achieved by the end of Phase II. ADA-positive samples from Phase II are required for assay development. In addition, isotyping (binding specificity) assays should be developed if requested by the regulatory authorities. Howard Hill (Huntingdon Life Sciences) followed with a presentation on LBA selectivity. He was philosophical in describing the selectivity of LBAs, labeling it 'the elephant in the room' and pointed out that, in some cases, comparison with chromatographic assays has demonstrated significant differences - always a potential headache. For example, we may need to pay more attention to protein fragments (metabolites). Mats Inganas (Gyros) made a presentation on the Gyrolab, a new platform that uses microfluidic principles for miniaturized immunoassay in CD format. The new format relies on flow-through immunoassay principles and offers much-reduced consumption of samples and reagents (nanoliter volumes) in reduced processing time and is fully automated. Rapid assay development is easily achievable and the versatility of the system makes it attractive for PK, ADA and biomarker studies. The last presentation in this section was delivered by Andreas Wolf (Micromet) who gave a fascinating insight into the development and potential application of bispecific T-cell-engager (BiTE) antibodies and their analysis. BiTE antibodies are single-chain bispecific antibody constructs with specificity for $\mathrm{CD} 3$ on T cells and a selected surface antigen on target cells. BiTE antibodies have been made to 16 antibody targets to date. Owing to the direct interaction between T-cells and cancer cells (oncology application), they are extremely potent. The potency results in extremely low steady-state plasma concentrations $(150 \mathrm{pg} / \mathrm{ml})$, which have proven challenging to quantitate, initially with a cell-based PK assay and more recently using an electrochemiluminescent assay which has been validated with a LLOQ of $20 \mathrm{pg} / \mathrm{ml}$.

\section{Unresolved issues in method validation}

Morten Rohde (Lundbeck) discussed issues with urine analyses from clinical studies. Urine is often considered an easy matrix compared with plasma or blood. In reality, although the sample preparation may be relatively simple, other factors bring complications, such as urine $\mathrm{pH}$, subject variability and insolubility caused 
by lack of solubilizing proteins in the matrix. It is very difficult to test all factors that may have an impact on the results. Nico van de Merbel (PRA International) continued on the same theme. He also highlighted potential issues such as $\mathrm{pH}$ variability and insolubility and, as a consequence, container adsorption and the potential need for additives, inhomogeneity of the sample, an abundance of metabolites (especially Phase II metabolites) and clinical sample collection and handling. As urine data are often of secondary importance, urine methodology should be fit-for-purpose (qualified) as opposed to fully validated.

Taha Rezai (Thermo) presented on the use of the TSQ Vantage ${ }^{\mathrm{TM}}$ for peptide quantitation with applications in the analyses of a panel of apolipoproteins and parathyroid hormone analysis. Massimo Breda (Accelera) and Ronald de Vries (Johnson and Johnson) both looked at tissue analysis. Massimo concluded that it is impossible to use spiked samples that mimic real samples and that full validation of tissue assays is not recommended. Ronald emphasized the need to adopt the correct homogenization approach and an extended recovery protocol, with key challenges being tissue stability assessment and the implementation of a tiered approach for tissue homogenate qualification, as opposed to full validation. Finally, Morten Kall (Lundbeck) described the evaluation of an isochronic study design for the long-term frozen storage stability of drugs in biological matrices (based on a method described elsewhere [1]). This is based on the assumption that the degradation of unstable compounds in biological matrices is negligible at temperatures lower than $-130^{\circ} \mathrm{C}$, all stability study data being referenced versus samples stored at $-150^{\circ} \mathrm{C}$. Results from 121 studies performed over the past 8 years suggest that, in all studies, stability is always fine at $-80^{\circ} \mathrm{C}$ but not always at $-20^{\circ} \mathrm{C}$, which also indicates that if stability is demonstrated at $-20^{\circ} \mathrm{C}$, there is also stability at $-80^{\circ} \mathrm{C}$. This proves that there is no issue in shipping samples on Cardice ${ }^{\circledR}$ for a short period, even if only $-20^{\circ} \mathrm{C}$ stability data is available.

\section{Summary}

This meeting has rapidly established itself on the conference circuit as the 'must-attend' meeting for the bioanalytical community and it is attracting increasing interest abroad. The venue and layout facilitated the quality of the presentations, discussions and networking opportunities. The 2010 meeting is already being planned by the enthusiastic and hard working Steering Committee. Please mark your calendars for 1-3 December 2010, Barcelona, Spain. The EBF looks forward to your contribution.

\section{Financial \& competing interests disclosure}

The author has no relevant affiliations or financial involvement with any organization or entity with a financial interest in or financial conflict with the subject matter or materials discussed in the manuscript. This includes employment, consultancies, honoraria, stock ownership or options, expert testimony, grants or patents received or pending, or royalties.

No writing assistance was utilized in the production of this manuscript.

\section{Bibliography}

1 Dadgar D, Burnett PE, Choc MG, Gallicano K, Hooper JW. Application issues in bioanalytical method validation, sample analysis and data reporting. J. Pharm. Biomed. Anal. 13(2), 89-97 (1995).

\section{Websites}

101 US FDA. Guidance for Industry: Bioanalytical Method Validation. Rockville, MD, USA: US Department of Health and Human Services, FDA, Center for Drug Evaluation and Research, 2001 www.fda.gov/downloads/Drugs/

GuidanceComplianceRegulatoryInformation/ Guidances/UCM070107.pdf

102 European Medicines Agency concept paper/ recommendations on the need for a (CHMP) guideline on the validation of bioanalytical methods. EMA/CHMP/EWP/531305/2008 www.ema.europa.eu/pdfs/human/ewp/ 53130508en.pdf

103 European Medicines Agency draft guideline on validation of bioanalytical methods www.ema.europe.eu/pdfs/human/ewp/19221709en. pdf

104 EBF and EUFEPS Workshop http://sites.google.com/site/emeabmv/

105 US FDA. Guidance for Industry: Safety Testing of Drug Metabolites www.fda.gov/downloads/drugs/ guidancecomplianceregulatoryinformation/ guidances/ucm079266.pdf\#6

106 Connecting strategies on dried blood spots http://sites.google.com/site/ebfdbs/ 